\title{
Comentarios sobre Harel y Teichman
}

\author{
Notes on Harel and Teichman*
}

Magdalena Małecka ${ }^{* *}$

Recepción: 27/05/2018

Evaluación: 23/08/2018

Aceptación final: 28/05/2019

\begin{abstract}
Resumen: Este texto es un comentario sobre el llamado de Harel y Teichman para una mayor "base empírica" del análisis económico-conductista del derecho. Aquí intento demostrar que el carácter normativo del derecho plantea un desafío serio, pero no discutido en el documento, para los estudios conductista y experimental del derecho. Discuto brevemente los puntos principales planteados por los autores y luego caracterizar de manera concisa el enfoque conductista de la ley que complementa su análisis. Después, discuto, sobre la base de dos trabajos experimentales tomados de otras ramas del derecho, los obstáculos para los estudios empíricos y experimentales del análisis económico-conductista del derecho. Concluyo con el breve comentario en la última sección del documento que se refiere a la posibilidad de sacar conclusiones normativas de las encuestas sobre las opiniones morales de las personas sobre el castigo.
\end{abstract}

Palabras clave: análisis económico-conductista del derecho, filosofía y metodología de la ciencia, ciencia jurídica empírica, poder expresivo del derecho

Traducción del inglés de Sofía Pezzano.

** PhD, Marie Skłodowska-Curie Fellow, Stanford University y University of Helsinki, Helsinki, Finlandia. Correo electrónico: magdalena.malecka@helsinki.fi. Este trabajo fue apoyado por la Academia de Finlandia [n. ${ }^{\circ}$ de subvención 308682] y por la Marie Skłodowska-Curie Fellowship EPISTEMEBEHAVIOUR, Comisión Europea. 
Magdalena Małecka

\begin{abstract}
This text is a commentary on Harel and Teichman's call for more "empirical grounding" of the behavioural analysis of law. I attempt to show that the normative character of law poses a serious, yet another and not discussed in the paper, challenge, for the behavioural and experimental studies of law. I briefly discuss the main points of raised by the authors and then I concisely characterize the behavioural approach to law that complements their analysis. Afterwards I discuss, on the basis of two experimental works taken from other branches of law, the obstacles to the empirical and experimental studies of the behavioural impact of law. I conclude with the brief remark on the last section of the paper that concerns the possibility of drawing normative conclusions from the surveys on people's moral views on punishment.
\end{abstract}

Keywords: behavioural law \& economics, philosophy and methodology of science, empirical legal science, expressive power of law

El artículo Análisis conductista del derecho penal y su aplicación: una amistosa revisión crítica de Alon Harel y Doron Teichman, analiza los importantes desafíos que implica la aplicación de la economía conductista al derecho. Tomando al derecho penal como ejemplo, los autores afirman que existe una incompatibilidad entre la comprensión del derecho como una empresa normativa, elaborada desde la teoría jurídica, y los modos en que, quienes proponen traer al derecho la investigación conductista, definen derecho (normas jurídicas) en sus análisis. He hecho un comentario similar en un artículo que escribí en colaboración con Robert Lepenies ${ }^{1}$, en el que afirmamos que la adopción generalizada de políticas públicas basadas en los resultados de investigaciones conductistas, está en contradicción y puede echar por tierra la función normativa del derecho. Los autores también discuten las fallas metodológicas del análisis conductista del derecho, que derivan de lo que ellos llaman el problema de la indeterminación. Según ellos, la indeterminación implica que las predicciones derivadas de la investigación conductista pueden ser contradictorias. Debido a los pro-

1 Lepenies, R. \& Małecka, M., "The Institutional Consequences of Nudging-Nudges, Politics, and the Law", Review of Philosophy and Psychology, 6 (3), 2015, p. 427-437. 
blemas señalados, Harel y Teichman exigen una mayor "base empírica" al análisis conductista del derecho. En respuesta a este reclamo, mi comentario intenta mostrar que el carácter normativo del derecho plantea otro serio desafío, no discutido en el artículo de Harel y Teichman, para los estudios conductistas y experimentales del derecho.

Parto de una caracterización concisa del enfoque conductista del derecho, que complementa la primera sección del artículo. Luego, basada en dos trabajos experimentales obtenidos de otras ramas del derecho, discuto los obstáculos a los estudios empíricos y experimentales del impacto conductista del derecho. En la última parte, concluyo con una breve observación sobre de la posibilidad de sacar conclusiones normativas a partir de investigaciones sobre los juicios morales de las personas acerca del castigo.

\section{Análisis económico-conductista del derecho: princi- pales tesis e hipótesis}

El enfoque conductista del análisis económico del derecho se basa en tres principios básicos. En primer lugar, los defensores de este enfoque critican al análisis económico del derecho neoclásico porque propone modelos del comportamiento humano altamente idealizados, que carecen de poder predictivo. Este tipo de críticas suele asociarse a críticas hacia la teoría de la decisión racional o la presunción de racionalidad. La principal objeción formulada por los teóricos conductistas, tiene que ver con la aceptación de asunciones poco realistas sobre el comportamiento humano y la toma de decisiones. Estas asunciones fracasan en predecir cómo actúan y deciden las personas, ya que es posible observar que en algunos contextos no se comportan "racionalmente", es decir, de acuerdo con las predicciones derivadas de los modelos neoclásicos. En segundo lugar, los especialistas en los enfoques conductistas proponen observar, analizar y explicar los comportamientos "reales" de la gente. Hacen énfasis en que muchas desviaciones de las predicciones basadas en modelos neoclásicos son sistemáticas y pueden ser explicadas sobre la base de una teoría alternativa (de la toma de decisiones). Proponen analizar las decisiones de las personas a partir de observaciones, en vez de formular hipótesis teóricas. Su ambición es pro- 
poner un modelo más realista del comportamiento en escenarios jurídicos, con mayor poder predictivo y explicativo. Finalmente, los defensores del enfoque conductista creen que sus análisis del comportamiento pueden y deberían ser utilizados por quienes crean las leyes y las políticas públicas. Ellos afirman que las intervenciones en la esfera decisoria de las personas deberían basarse en el conocimiento acerca de las posibles respuestas conductistas al derecho. En otras palabras, proponen aplicar los resultados de las ciencias conductistas para proponer mejores y más realistas recomendaciones de políticas públicas; especialmente en la medida en que el enfoque neoclásico, en su opinión, se ha vuelto menos relevante para la elaboración de políticas jurídicas².

Los defensores del enfoque conductista, proponen basar el análisis económico del derecho en presunciones diferentes acerca del comportamiento, fundadas en observaciones de cómo se comportan las personas. En este enfoque se acentúa la importancia de los "límites" (racionalidad limitada, fuerza de voluntad limitada y auto-interés limitado). Además, se afirma que las desviaciones de los modelos neoclásicos tienen un carácter sistemático y, por esa razón, son predecibles. El análisis económico-conductista del derecho se ha inspirado en gran medida en la concepción de la heurística de Kahneman y Tversky, así como en su teoría prospectiva. Esta teoría se utiliza para explicar cómo la forma en que se presenta un problema a un sujeto influye en sus decisiones, así como también para predecir conductas de aversión o de búsqueda de riesgo ${ }^{3}$. La concepción de la heurística, por otro lado, esclarece la utilización de atajos mentales durante los procesos de evaluación y toma de decisiones en entornos jurídicos ${ }^{4}$. Además, los autores conductistas a menudo se refieren a la investigación sobre normas sociales, argumentando que las personas no se comportan de acuerdo con las predicciones de los modelos neoclásicos porque no siempre maximizan la utilidad (entendida como auto-interés). El hecho de que la gente siga

2 Korobkin, R. \& Ulen, T., "Law and Behavioral Science: Removing the Rationality Assumption from Law and Economics", California Law Review, 88 (4), 2000, p. 1056.

3 Véase Kahneman, D. \& Tversky, A., "Prospect theory: an analysis of decision under risk", Econometrica, 47 (2), 1979, pp. 263-291.

4 Véase Tversky, A. \& Kahneman, D., "Judgment Under Uncertainty: Heuristics and Biases", Science, New Series, 185 (4157), 1974, pp. 1124-1131. 
normas sociales (a veces en contra de sus intereses a corto plazo) se considera un factor importante que influye en el comportamiento.

\section{El desafío de estudiar normas en escenarios experimentales}

A continuación se analizan distintos experimentos que examinan la influencia directa del derecho, esto es, una reacción conductista a la introducción de una norma jurídica (simulada en un contexto experimental). Esos experimentos, precisamente, abordan el impacto conductual del derecho, que, según afirman, será explicado por los especialistas en el análisis conductista del derecho. Mi objetivo es analizar cómo se simulan las normas jurídicas y cómo se trata el aspecto normativo de las normas jurídicas en contextos experimentales, así como también la forma en que se interpretan los resultados. Me pregunto si, y en su caso, en qué medida se pueden extraer conclusiones sobre la influencia de las normas jurídicas a partir de los resultados obtenidos durante esos experimentos.

La afirmación de que las normas jurídicas no solo tienen un efecto disuasorio o, en términos más generales, de incentivo, sino que también pueden influir en el comportamiento a través de la expresión de valores sociales, llevó a algunos estudiosos del análisis económico del derecho a proponer un nuevo acercamiento entre ambas ramas: el análisis económico-expresivo del derecho. Este enfoque se basa en la idea de que el derecho expresa valores sociales, gracias a los cuales puede crear puntos de convergencia que ayuden a coordinar el comportamiento de las personas. Quienes proponen este enfoque distinguen entre el aspecto de obligación y el aspecto de incentivo de las normas jurídicas, y sostienen que las obligaciones "afectan el comportamiento en un modo específico" 5 . Roberto Galbiati y Pietro Vertova han diseñado experimentos en los cuales han testeado la influencia de las normas jurídicas sobre el comportamiento. Ellos definen

5 Cooter, R., “Expressive Law and Economics”, Journal of Legal Studies, 27 (2), 1998, p. 587. 
a las normas jurídicas, a las que llaman "reglas formales", como "obligaciones respaldadas por incentivos", refiriéndose a la definición de normas jurídicas que denominan positivista. De esta manera, ensanchan la definición ampliamente aceptada dentro de los estudios del análisis económico del derecho, que identifica las normas jurídicas con incentivos. Galbiati y Vertova subrayan la importancia de distinguir el aspecto de incentivo de la norma jurídica de su componente normativo. "La parte de la obligación de la regla formal consiste en el contenido normativo establecido por la regla o, en otras palabras, en el comportamiento que la regla establece que las personas deberían seguir"7. En sus experimentos intentan separar el componente normativo del efecto de incentivo de la norma jurídica. A continuación me refiero a un experimento descrito e interpretado en el artículo Cómo afecta el derecho al comportamiento: obligaciones, incentivos y comportamiento cooperativo con el fin de presentar la forma en que su objetivo es alcanzado ${ }^{8}$.

El experimento es diseñado para probar la influencia de las reglas jurídicas en el comportamiento cooperativo durante el juego de bienes públicos. "A fin de comprender cómo las reglas formales afectan el comportamiento, resulta crucial estudiar experimentalmente cómo funcionan las obligaciones y cómo interactúan con los incentivos, influyendo en el comportamiento humano" 9 . En el experimento, los participantes fueron dotados de una cantidad inicial de fichas y se les pidió que tomaran dos decisiones: "contribución incondicional" (se solicitó a los sujetos contribuir al bien público) y "contribución condicional" (se solicitó a los sujetos elegir en qué cantidad contribuir al bien público en comparación con las diferentes contribuciones promedio de otros miembros del grupo). Luego se les preguntó sobre sus creencias acerca de las contribuciones incondicionales de los demás. Una contribución mínima fue fijada exógenamente por los experimentadores. Las opciones condicionales e incondicionales se diseñaron para testear cuáles eran los canales a través de los cuales los

6 Galbiati, R. \& Vertova, P., "How law affects behavior: Obligations, incentives and cooperative behavior", International Review of Law and Economics, 38, 2014, p. 2.

7 Ibidem, p. 2.

8 Ídem. 
incentivos y las obligaciones afectan el comportamiento (a través de creencias o preferencias, o ambas). Además, hubo dos variantes del juego (una sin incentivos y otra con una estructura de incentivos) en seis procesos experimentales diferentes: dos sin incentivos, tres con la misma estructura de incentivos y uno con un nivel muy bajo de incentivos. Estas variantes deberían haber testeado la forma en que las variaciones de las obligaciones y las variaciones de los incentivos afectan al comportamiento cooperativo, así como la manera en que las obligaciones y los incentivos interactúan entre sí. Los investigadores simularon una serie de experimentos de decisión única con bienes públicos. Las obligaciones estaban representadas por contribuciones mínimas no vinculantes al bien público exigidas en las instrucciones, los incentivos estaban representados por castigos probabilísticos por contribuciones por debajo del mínimo y recompensas probabilísticas por contribuciones por encima del mínimo (el seguimiento de las contribuciones también era probabilístico). Los esquemas de incentivos fueron estructurados de tal modo que no contribuir al bien público es la estrategia dominante para alguien que maximiza la utilidad.

La idea de aislar los efectos de incentivo y de obligación de las normas jurídicas se llevó a cabo de la siguiente manera: las obligaciones sin incentivos se simularon como un requisito de contribución por un monto del 80 por ciento de la dotación de fichas (sin recompensas/castigos). Los incentivos sin obligaciones se simularon como un requisito de contribución ( $s i n$ especificación de cantidad), pero acompañado por una estructura de monitoreo (un participante es recompensado si la contribución supera 0 , de lo contrario es penalizado, con la probabilidad de monitoreo siendo 1/12). Por el otro lado, las obligaciones con incentivos se simularon en tratamientos con una estructura de monitoreo y exigencia de contribución de una cantidad específica de fichas, que variaba entre 20 y 80 por ciento de dotación. También fue fijada cerca a cero en una de las versiones. Las variaciones en la cantidad de fichas permitieron a los investigadores mantener los incentivos marginales fijados a través de los tratamientos y hacerlos independientes de la contribución mínima (obligaciones). Por lo tanto, si se observaba alguna diferencia en los tratamientos con estructura de monitoreo, podría haberse imputado al efecto de la obligación sobre el beneficio. 
Galbiati y Vertova observaron, en primer lugar, que la introducción de una obligación en ausencia de incentivos llevaba a un aumento en la provisión del bien público; en segundo lugar, que la introducción de incentivos sin una obligación no afectaba significativamente las contribuciones, mientras que, en tercer lugar, cuando las obligaciones y los incentivos se combinaban, se reforzaba fuertemente la cooperación. Cuando interpretaron los resultados experimentales, llegaron a la conclusión de que las obligaciones tienen una influencia significativa en el comportamiento cooperativo, incluso si no son respaldadas por incentivos. Si los incentivos están vinculados a obligaciones, entonces las variaciones en las obligaciones afectan al comportamiento, incluso si los incentivos se mantienen constantes. Sin embargo, la introducción de incentivos a la exigencia de contribución refuerza considerablemente la cooperación. Los autores opinan que el contenido de una norma es percibido como una obligación si está respaldada por incentivos. En ese caso, el papel principal de los incentivos es más bien dar prominencia al contenido de las reglas y no tanto modificar los beneficios (como sostienen los estudiosos del análisis económico del derecho neoclásico). Galbiati y Vertova también concluyeron que el efecto de las obligaciones sobre el comportamiento depende, por un lado, de sus impactos en las creencias de las personas sobre las contribuciones de los demás y, por otro lado, de sus preferencias por la cooperación.

Cabe destacar que, en los experimentos, las tareas se comunicaban a los participantes en un lenguaje que no se parecía al lenguaje jurídico. Se solicitó a los participantes contribuciones monetarias. Puede reflexionarse acerca de si tal simulación de escenarios jurídicos en una situación experimental es adecuada y en qué medida los resultados de los experimentos pueden extrapolarse de un contexto experimental a uno jurídico. En la siguiente sección presento experimentos en los cuales los autores intentan simular normas jurídicas de manera diferente, es decir, mediante una forma lingüística. La crítica más seria se refiere al hecho de que Galbiati y Vertova identifican el aspecto normativo de las reglas jurídicas con su aspecto expresivo (también llamado "poder expresivo de las reglas jurídicas"). Esto significa que ellos identifican las normas jurídicas con normas sociales y morales, y que tratan la normatividad de esas normas como equivalentes. En otras palabras, los autores parecen asumir que no hay 
nada específico los modos de, y las razones para, seguir normas jurídicas, sociales o morales.

Los experimentos conducidos por Lewis Kornhauser y Andrew Schotter tenían por objeto testear los efectos de incentivo de las reglas de responsabilidad en materia de responsabilidad civil (derecho de daños). Sin embargo, en una versión de los experimentos examinaron el impacto expresivo del derecho. Puesto que escogieron un camino que difiere del elegido por Galbiati y Vertova para simular obligaciones (y que además dio lugar a resultados desconcertantes), decidí mencionar su experimento en este trabajo.

Kornhauser y Schotter comienzan el artículo, que presenta los resultados de sus experimentos, diciendo que "durante los últimos veinticinco años, el debate jurídico sobre la cuestión empírica de la disuasión, en general, no ha girado en torno a los efectos disuasorios reales, sino en torno a modelos económicos del derecho de daños que predicen el efecto disuasorio de diversas normas jurídicas" ${ }^{10}$. Los autores, principalmente, están interesados en testear si el nivel de diligencia y el nivel de actividad que las personas eligen en entornos experimentales se corresponden con el que surge de las predicciones hechas a partir de los modelos del análisis económico del derecho neoclásico. Testean un modelo de accidente de un único actor en el cual las acciones de un individuo influyen en la frecuencia con la que se espera que ocurra una pérdida. El accidente se caracteriza como una pérdida, y las normas jurídicas se caracterizan como reglas que determinan quién debe soportar la pérdida (la víctima o el causante). Las hipótesis basadas en el modelo teórico del análisis económico del derecho son las siguientes:

1) El nivel de diligencia adoptado por un causante potencial bajo la regla de responsabilidad objetiva es idéntico al nivel de diligencia adoptado bajo la regla de responsabilidad subjetiva, si esta última fija el nivel de debido cuidado en un nivel socialmente óptimo.

${ }^{10}$ Kornhauser, L. \& Schotter, A., "An experimental study of single-actor accidents", Journal of Legal Studies, 19, 1990, p. 203. 
2) Un individuo potencialmente involucrado en un accidente (ya sea causante o víctima) adherirá al estándar de diligencia si este se fija ligeramente por encima del nivel socialmente óptimo, pero lo abandonará si se fija significativamente por encima de ese nivel.

3) El nivel de actividad bajo una regla de responsabilidad subjetiva con el estándar de debido cuidado establecido en el nivel óptimo de diligencia, supera el nivel de actividad bajo la regla de responsabilidad objetiva ${ }^{11}$.

Los autores también decidieron probar "la fuerza normativa (moral) de las normas jurídicas" ${ }^{12}$. Por lo tanto, formularon una hipótesis adicional que afirma que las personas tienen una tendencia a adherir a estándares de diligencia aun si este es fijado significativamente por encima del nivel socialmente óptimo.

Se les informó a los participantes que estaban involucrados en un experimento de toma de decisiones que duraría 35 períodos (etapas). Se les entregó un monto inicial de dinero y en cada etapa se les solicitó que eligieran un "número de decisión" (entre 1 y 100), con las siguientes consecuencias: un cierto costo por "número de decisión" y una determinada influencia del "número de decisión" en la probabilidad de acaecimiento de un "evento". El acaecimiento de ese "evento" también tenía un costo, determinado por los experimentadores. En las condiciones del régimen de responsabilidad objetiva, los participantes debían pagar un determinado costo siempre que ocurría un "evento". Bajo el régimen de responsabilidad subjetiva, debían pagar solamente si elegían un "número de decisión" por debajo del llamado "número crítico". Se suponía que el "número de decisión" simulaba un nivel de diligencia, el "evento" un accidente y el "número crítico", un estándar legal de diligencia. Se hicieron diez versiones del experimento (con 35 etapas cada una).

No describo todas las versiones del experimento en detalle, ya que son bastante técnicas, pues mi objetivo aquí es más bien comentar el intento mismo de testear el impacto expresivo y normativo del derecho. En general, 
Kornhauser y Schotter han observado que bajo un sistema de responsabilidad objetiva los sujetos muestran un nivel excesivo de diligencia y, luego de varias etapas, reducen el nivel de diligencia por debajo del nivel óptimo. Sin embargo, en un sistema de responsabilidad subjetiva, se comportan de acuerdo con las predicciones basadas en el modelo del análisis económico del derecho. Así, Kornhauser y Schotter concluyeron que "bajo el régimen de responsabilidad objetiva los sujetos carecen de orientación sobre cómo abordar el problema de decisión que se les presenta. Comienzan la búsqueda de soluciones en niveles excesivamente altos de diligencia y luego reducen sus niveles de diligencia por debajo del óptimo (...). Bajo una norma de responsabilidad subjetiva, el estándar de diligencia pone un límite más alto en la búsqueda de soluciones de los sujetos. Cuando el estándar es excesivamente alto, los sujetos de alguna manera aprenden que deberían abandonarlo"13.

Con el fin de testear el impacto normativo y expresivo del derecho, cambiaron el lenguaje de las instrucciones de tal manera que usaron terminología similar a la jurídica, y lo hicieron en dos versiones del experimento (bajo escenarios de responsabilidad objetiva y subjetiva). En otras palabras, la formulación de las instrucciones fue la misma, con excepción de los términos: "número de decisión", "evento" y "número crítico", que fueron reemplazados por "nivel de diligencia", "accidente" y "estándares legales de diligencia" respectivamente. Curiosamente, si el nivel de diligencia era fijado por los experimentadores por encima de un nivel socialmente óptimo, las personas tendían a exhibir niveles significativamente más bajos de diligencia que en la versión del experimento sin el lenguaje "normativo". Cuando fue fijado en un nivel socialmente óptimo, no se observó ningún cambio en el cumplimiento. Los autores etiquetaron aquel resultado como "paradójico" y se resignaron a seguir investigando los efectos normativos del derecho. Sin embargo, si las personas se comportan de manera diferente en estos dos escenarios, puede significar que hay un factor que es responsable de esa diferencia. Dado que las personas se comportan de una manera que no confirma la hipótesis formulada por los investigadores, tal resultado puede interpretarse como evidencia de la importancia del factor normativo para las decisiones tomadas por los sujetos. Más especí- 
ficamente, puede significar que el factor "jurídico" tiene un impacto más fuerte que el factor "económico" o "racional". Esto podría ser evidencia de que el aspecto normativo o "jurídico" del derecho (esto es, el hecho de que una conducta es exigida por la ley) tiene un impacto conductual único que requiere clarificación.

Los test experimentales de accidentes con un único actor, demuestran que existen dificultades para simular contextos y normas jurídicas en escenarios experimentales. Los problemas de decisión, en las versiones que testeaban los efectos de incentivo de las normas de responsabilidad, se sustituyeron durante los experimentos por decisiones basadas en números. Los investigadores esperaban que los sujetos decidieran sobre la base de cálculos realizados en cada ronda del experimento. Un diseño de este tipo suscita interrogantes sobre bajo qué condiciones las decisiones sobre números pueden ser extrapoladas a las decisiones de personas bajo el derecho de daños. Lewis y Kornahuser extrapolaron sus hallazgos sin las debidas reservas.

Otro problema relacionado con la cuestión de la extrapolación, evocado por el experimento de Lewis y Kornhauser, así como también por el estudio de Galbiati y Vertova, es la simulación experimental de normas jurídicas. Galbiati y Vertova formularon las instrucciones sin utilizar terminología jurídica. Sus instrucciones simplemente tenían la forma de un pedido o una solicitud de pagar un monto determinado de dinero para financiar un bien público, pero en ningún momento sugirieron que los sujetos estuviesen obligados a hacerlo o que su decisión pudiera tener algo que ver con el derecho. Lewis y Kornhauser, por otro lado, decidieron testear el impacto expresivo de las reglas de responsabilidad a través de la modificación del lenguaje de las instrucciones, de manera tal que incluyera terminología jurídica. Como consecuencia, arribaron a un resultado, en sus palabras, paradójico: los participantes de los experimentos se comportaron de una manera que los autores interpretaron como "en contra del auto interés" y "menos diligentemente" que en las versiones con "incentivos". Este resultado puede ser discutido de dos maneras.

En primer lugar, puede sugerir que en algunos contextos las personas se comportan en contra de su interés si se enfrentan con normas jurídicas. En el experimento, las personas cambiaron su comportamiento de uno auto-interesado a uno contrario a su auto-interés, luego de que las instruc- 
ciones cambiaran de la versión de los "incentivos" a la versión "normativa”, mientras que las demás condiciones se mantuvieron constantes. Esto significa que las normas jurídicas pueden cambiar el comportamiento de una forma que no puede ser explicada basándonos en la teoría económica o de la decisión. En segundo lugar, el experimento de Lewis y Kornhauser es relevante para el problema de la expresión lingüística de las normas jurídicas en escenarios experimentales. Las normas jurídicas se formulan de distintas maneras en actos jurídicos: como proposición deóntica, como descripción de comportamientos, o como la denominada "forma paradigmática de la norma jurídica”. Todo investigador interesado en la simulación de las normas jurídicas a través de la formulación lingüística, debe decidir cuál formulación elegir. También debería considerar si esas expresiones lingüísticas pueden conducir a resultados conductuales divergentes. En caso afirmativo, debe proporcionarse una interpretación de esa observación.

Los experimentos descritos anteriormente, se ocupan de testear el aspecto normativo de las normas jurídicas. Notablemente, los autores identificaron el aspecto normativo con el expresivo (la expresión de valores). El resultado de esto es la asimilación de las normas jurídicas con los valores y normas sociales, así como también la reducción del componente normativo (jurídico) al aspecto social o ético. Sin embargo, los mencionados autores no dan ninguna justificación para esa reducción. Por otra parte, de esa manera tocan el viejo debate filosófico sobre los factores que hacen que el derecho sea normativo. A través de la identificación de los aspectos normativos con el poder expresivo del derecho, desatienden la tradición positivista y se ubican a sí mismos no solo del lado de los realistas jurídicos, sino también cerca de los defensores de las doctrinas del derecho natural. Esto, sin embargo, involucra sus análisis en complejas discusiones filosóficas.

\section{Las intuiciones morales de las personas y la teoría científica}

En la última parte de su artículo, Harel y Teichman critican la idea de basarse en estudios empíricos, acerca de juicios morales sobre el contenido del derecho penal, para diseñar el derecho penal. Se suele afirmar que si los 
requerimientos del derecho se corresponden con las intuiciones morales de las personas, entonces sería más probable que las personas obedezcan el derecho y, por lo tanto, para que el derecho penal efectivamente impacte en el comportamiento debería expresar los juicios morales de sus destinatarios. Más arriba he discutido los problemas metodológicos relacionados con los estudios sobre el poder expresivo de las normas jurídicas. Harel y Teichman también señalan que los intentos de ajustar el contenido del derecho penal a las intuiciones morales de las personas es problemático, ya que trata esas intuiciones, así como las opiniones sobre las acciones centradas en la disuasión, como si fueran dadas (naturales). Asimismo, me gustaría agregar, coincidiendo con las críticas de los autores, que un enfoque de esa índole presupone que las opiniones de las personas sobre la sociedad, incluidas sus opiniones sobre lo que es justo, no están sujetas a la influencia del trabajo científico. Los filósofos de las ciencias sociales, sin embargo, señalan el fenómeno de cómo la comprensión que las personas tienen de diferentes ámbitos de la realidad social, cambia bajo la influencia de, y en respuesta a, las teorías científicas sociales de esas áreas. Este fenómeno fue llamado reflexividad y fue muy discutido por los estudiosos que trabajan en distintas áreas de las ciencias sociales ${ }^{14}$. Por lo tanto, la percepción del derecho como un incentivo o estímulo, y no como una norma o expresión de juicios morales, puede cambiar si los enfoques económicos o conductistas del derecho se difunden en el mundo académico y más allá. Esto invalidaría la idea de diseñar una ley ajustándola a los juicios morales de las personas.

\section{Bibliografía}

Cooter, R., "Expressive Law and Economics", Journal of Legal Studies, 27 (2), 1998, pp. 55-79.

${ }^{14}$ Véase Merton, R. K., “The self-fulfilling prophecy”, The Antioch Review, 8 (2), 1948, pp. 193 210; Woolgar, S. (ed.), Knowledge and Reflexivity: New Frontiers in the Sociology of Knowledge Sage Publications, Thousand Oaks, CA, 1988 y Soros, G., "Fallibility, reflexivity and the human uncertainty principle", Journal of Economic Methodology, 20, 2013, pp. 309-329. 
Galbiati, R. \& Vertova, P., "How law affects behavior: Obligations, incentives and cooperative behavior", International Review of Law and Economics, 38, 2014, pp. 48-57.

Kahneman, D. \& Tversky, A., "Prospect theory: an analysis of decision under risk", Econometrica, 47 (2), 1979, pp. 263-291.

Kornhauser, L. \& Schotter, A., "An experimental study of single-actor accidents", Journal of Legal Studies, 19, 1990, pp. 203-233.

Korobkin, R. \& Ulen, T., "Law and Behavioral Science: Removing the Rationality Assumption from Law and Economics", California Law Review, 88 (4), 2000, pp. 1051-1144.

Lepenies, R. \& Małecka, M., “The Institutional Consequences of NudgingNudges, Politics, and the Law", Review of Philosophy and Psychology, 6 (3), 2015, pp. 427-437.

Merton, R.K., "The self-fulfilling prophecy", The Antioch Review, 8 (2), 1948, pp. 193-210.

Soros, G., "Fallibility, reflexivity and the human uncertainty principle", Journal of Economic Methodology, 20, 2013, pp. 309-329.

Tversky, A. \& Kahneman, D., "Judgment Under Uncertainty: Heuristics and Biases", Science, New Series, 185, 4157, 1974, pp. 1124-1131.

Woolgar, S. (ed.), Knowledge and Reflexivity: New Frontiers in the Sociology of Knowledge; Thousand Oaks, CA, Sage Publications, 1988. 